\title{
Representations of 3-Dimensional Simple Multiplicative Hom-Lie Algebras
}

\author{
Xiuxian Li \\ Department of Mathematics, Tongji University, Shanghai 200092, China \\ Correspondence should be addressed to Xiuxian Li; lxxcaptain@126.com
}

Received 6 July 2013; Accepted 23 September 2013

Academic Editor: Wen-Xiu Ma

Copyright (C) 2013 Xiuxian Li. This is an open access article distributed under the Creative Commons Attribution License, which permits unrestricted use, distribution, and reproduction in any medium, provided the original work is properly cited.

\begin{abstract}
The aim of this paper is to study representations of 3 -dimensional simple multiplicative Hom-Lie algebras $(\mathfrak{g} ;[\cdot, \cdot] ; \alpha)($ whose structure is of $A_{1}$-type). In this paper we can see that a finite dimensional representation of $(\mathfrak{g} ;[\cdot, \cdot] ; \alpha)$ is not always completely reducible, and a representation of $(\mathfrak{g} ;[\cdot, \cdot] ; \alpha)$ is irreducible if and only if it is a regular Lie-type representation.
\end{abstract}

\section{Introduction}

In 2006, Hartwig, Larsson, and Silvestrov introduced the notion of a Hom-Lie algebra [1], which is a generalization of the notion of a Lie algebra. In particular, if $\alpha=$ id, then a Hom-Lie algebra is exactly a Lie algebra.

Because the Hom-Lie algebras are closely related to discrete and deformed vector fields, differential calculus [2, $3]$, and mathematical physics $[4,5]$, the Hom-Lie algebras have attracted more and more attention and become an active topic in recent years [6-8].

The representation theory plays an important role in Lie theory [9-11]. By means of the representation theory, we would be more aware of the corresponding algebras. Thus it is meaningful to obtain more information about the representations of Hom-Lie algebras.

In [7] the author defined the representations of HomLie algebras and the corresponding Hom-cochain complexes, and studied the cohomologies associated with the adjoint representation and the trivial representation. As is known, specific calculations about the representations of Hom-Lie algebras are still not solved. The diversity of the twist map of $\mathfrak{g}$ makes this topic interesting and complicated.

Thanks to the relationship between multiplicative HomLie algebras with $\alpha$ invertible and Lie algebras (Lemma 3), the representation theory of Lie algebras can be a reference to what is considered. The representation of a 3-dimensional simple Lie algebra plays a crucial role in the representation theory of semisimple Lie algebras over $\mathbb{C}[9]$. By the same reason, in this paper, we study the representations of 3dimensional simple multiplicative Hom-Lie algebras.

The paper is organized as follows. In Section 2 we study the structures of 3-dimensional simple multiplicative HomLie algebra and show that 3-dimensional simple multiplicative Hom-Lie algebras are of $A_{1}$-type. In Section 3, the representation $(\rho, V, \beta)$ of a multiplicative Hom-Lie algebra with $\alpha$ invertible is investigated and shows that when $\beta$ is invertible, $(\rho, V, \beta)$ is of Lie-type, which makes it convenient to study representations of multiplicative Hom-Lie algebras. In Section 4, we study regular Lie-type representations of 3-dimensional simple multiplicative Hom-Lie algebras and reflect the existence and irreducibility of representations of this type. In Section 5, we work over finite dimensional representations of 3-dimensional simple multiplicative HomLie algebras $(\mathfrak{g},[\cdot, \cdot], \alpha)$. In this section we can see that a finite dimensional representation of $(\mathfrak{g},[\cdot, \cdot], \alpha)$ is not always completely reducible and a representation of $(\mathfrak{g},[\cdot, \cdot], \alpha)$ is irreducible if and only if it is a regular Lie-type one.

Throughout this paper, unless otherwise stated, all algebras are finite dimensional and over the complex field $\mathbb{C}$.

\section{The Structures of 3-Dimensional Simple Multiplicative Hom-Lie Algebras}

First we give some important definitions on Hom-Lie algebras. 
Definition 1 (see [1]). A Hom-Lie algebra is a triple $(\mathfrak{g},[\cdot, \cdot], \alpha)$ consisting of a vector space $\mathfrak{g}$ over $\mathbb{C}$, a linear sef-map $\alpha$, and a bilinear map $[\cdot, \cdot]: \mathfrak{g} \times \mathfrak{g} \rightarrow \mathfrak{g}$ such that

$$
\begin{gathered}
{[x, y]=-[y, x], \quad \forall x, y \in \mathfrak{g},} \\
{[\alpha(x),[y, z]]+[\alpha(y),[z, x]]+[\alpha(z),[x, y]]=0,} \\
\forall x, y, z \in \mathfrak{g} .
\end{gathered}
$$

A Hom-Lie algebra $(\mathfrak{g},[\cdot, \cdot], \alpha)$ is said to be multiplicative if $\alpha([x, y])=[\alpha(x), \alpha(y)]$ for any $x, y \in \mathfrak{g}$; see [7].

Definition 2. Let $(\mathfrak{g},[\cdot, \cdot], \alpha)$ be a Hom-Lie algebra. If there exists a Lie algebra $\left(\mathfrak{g},[\cdot, \cdot]^{\prime}\right)$ such that $[x, y]=\alpha\left([x, y]^{\prime}\right)=$ $[\alpha(x), \alpha(y)]^{\prime}$, for all $x, y \in \mathfrak{g}$, then $(\mathfrak{g},[\cdot, \cdot], \alpha)$ is said to be of Lie-type, and $\left(\mathfrak{g},[\cdot, \cdot]^{\prime}\right)$ is called the compatible Lie algebra of $(\mathfrak{g},[\cdot, \cdot], \alpha)$. Furthermore, if the compatible Lie algebra of $(\mathfrak{g},[\cdot, \cdot], \alpha)$ is an $A$ (or $B, C, D)$-type Lie algebra, one calls $(\mathfrak{g},[\cdot, \cdot], \alpha)$ an $A$ (or $B, C, D$ )-type Hom-Lie algebra.

A subspace $\mathfrak{g}_{1}$ of $\mathfrak{g}$ is called an ideal of $(\mathfrak{g},[\cdot, \cdot], \alpha)$ if $\alpha\left(\mathfrak{g}_{1}\right) \subseteq$ $\mathfrak{g}_{1},\left[\mathfrak{g}_{1}, \mathfrak{g}\right] \subseteq \mathfrak{g}_{1}$ are satisfied. A center of $(\mathfrak{g},[\cdot, \cdot], \alpha)$ is defined as

$$
C(\mathfrak{g})=\{x \in \mathfrak{g} \mid[x, \mathfrak{g}]=0 ;[\alpha(x), \mathfrak{g}]=0\}
$$

A Hom-Lie algebra $(\mathfrak{g},[\cdot, \cdot], \alpha)$ is called simple if it has no nontrivial ideals and $[\mathfrak{g}, \mathfrak{g}]=\mathfrak{g}$.

Lemma 3. Let $(\mathfrak{g},[\cdot, \cdot], \alpha)$ be a multiplicative Hom-Lie algebra with $\alpha$ invertible. Then $(\mathfrak{g},[\cdot, \cdot], \alpha)$ is Lie-type with the compatible Lie algebra $\left(\mathfrak{g},[\cdot, \cdot]^{\prime}\right)$, where $[\cdot, \cdot]^{\prime}$ is defined by $[x, y]^{\prime}=$ $\alpha^{-1}([x, y])$, for all $x, y \in \mathfrak{g}$.

Proof. Let $[x, y]^{\prime}=\alpha^{-1}([x, y])$ for any $x, y \in \mathfrak{g}$. Since $(\mathfrak{g},[\cdot, \cdot], \alpha)$ is multiplicative, we have

$$
\begin{aligned}
\alpha([x, y]) & =[\alpha(x), \alpha(y)] \Longrightarrow \alpha^{2}\left([x, y]^{\prime}\right) \\
& =\alpha\left([\alpha(x), \alpha(y)]^{\prime}\right)
\end{aligned}
$$

and thus $\alpha\left([x, y]^{\prime}\right)=[\alpha(x), \alpha(y)]^{\prime}$ follows.

In the following we will show that $\left(\mathfrak{g},[\cdot, \cdot]^{\prime}\right)$ is a Lie algebra. First it is obvious that $[\cdot, \cdot]^{\prime}$ is skew-symmetric. Next $\forall x, y, z \in \mathfrak{g}$,

$$
\begin{aligned}
& \mho_{x, y, z}\left[x,[y, z]^{\prime}\right]^{\prime} \\
& =\mho_{x, y, z} \alpha^{-1}\left[x, \alpha^{-1}([y, z])\right] \\
& =\circlearrowleft_{x, y, z} \alpha^{-1}\left[\alpha^{-1} \alpha(x), \alpha^{-1}([y, z])\right] \\
& =\mho_{x, y, z} \alpha^{-2}([\alpha(x),[y, z]])=0,
\end{aligned}
$$

where $\sigma_{x, y, z}$ denotes a summation over the cyclic permutation on $x, y, z$. Now it follows that $\left(\mathfrak{g},[\cdot, \cdot]^{\prime}\right)$ is a Lie algebra.
Theorem 4. Let $(\mathfrak{g},[\cdot, \cdot], \alpha)$ be a 3-dimensional simple multiplicative Hom-Lie algebra; then $(\mathfrak{g},[\cdot, \cdot], \alpha)$ is $A_{1}$-type and

$$
\begin{aligned}
& (\mathfrak{g},[\cdot, \cdot], \alpha) \\
& \quad=\left\{h, e, f \mid[h, e]=2 a e,[h, f]=-2 a^{-1} f,[e, f]=h\right\}, \\
& \alpha(h, e, f)=(h, e, f)\left(\begin{array}{ccc}
1 & 0 & 0 \\
0 & a & 0 \\
0 & 0 & a^{-1}
\end{array}\right)_{0 \neq a \in \mathbb{C}} .
\end{aligned}
$$

Proof. If $\operatorname{Ker}(\alpha) \neq 0$, then $\alpha(\operatorname{Ker}(\alpha))=0, \alpha([\mathfrak{g}, \operatorname{Ker}(\alpha)])=$ 0 ; that is, $\operatorname{Ker}(\alpha)$ is a nontrivial ideal of $(\mathfrak{g},[\cdot, \cdot], \alpha)$, which is a contradiction to the simplicity of $(\mathfrak{g},[\cdot, \cdot], \alpha)$. So $\alpha$ is invertible. Now by Lemma 3 , we have that $(\mathfrak{g},[\cdot, \cdot], \alpha)$ is Lietype with the 3-dimensional Hom-Lie admissible algebra $\left(\mathfrak{g},[\cdot, \cdot]^{\prime}\right)=\left(\mathfrak{g}, \alpha^{-1}([\cdot, \cdot])\right)$.

If $\left(\mathfrak{g},[\cdot, \cdot]^{\prime}\right)$ is an abelian Lie algebra, then we can deduce that $(\mathfrak{g},[\cdot, \cdot], \alpha)$ is also abelian, which is absurd.

Suppose that $\left(\mathfrak{g},[\cdot, \cdot]^{\prime}\right)$ has a 1-dimensional center $\mathbb{C} x$. Note that $[x, \mathfrak{g}]=\alpha\left([x, \mathfrak{g}]^{\prime}\right)=0$, and for all $y \in \mathfrak{g}, \exists y^{\prime} \in \mathfrak{g}$ such that $y=\alpha\left(y^{\prime}\right)$, we have

$$
[\alpha(x), y]=\left[\alpha(x), \alpha\left(y^{\prime}\right)\right]=\alpha\left(\left[x, y^{\prime}\right]\right)=0 .
$$

That is, $(\mathfrak{g},[\cdot, \cdot], \alpha)$ has a 1 -dimensional center $\mathbb{C} x$, which is a contradiction to the simplicity of $(\mathfrak{g},[\cdot, \cdot], \alpha)$.

If $[\mathfrak{g}, \mathfrak{g}]^{\prime} \neq \mathfrak{g}$, then

$$
[\mathfrak{g}, \mathfrak{g}]=\alpha^{-1}\left([\mathfrak{g}, \mathfrak{g}]^{\prime}\right)=\left[\alpha^{-1}(\mathfrak{g}), \alpha^{-1}(\mathfrak{g})\right]^{\prime}=[\mathfrak{g}, \mathfrak{g}]^{\prime} \neq \mathfrak{g}
$$

which is impossible.

Now we can get that $[\mathfrak{g}, \mathfrak{g}]^{\prime}=\mathfrak{g}$. By Lie theory, $\left(\mathfrak{g},[\cdot, \cdot]^{\prime}\right)$ is an $A_{1}$-type Lie algebra with a basis $\{h, e, f\}$ and a bracket $[h, e]^{\prime}=2 e,[h, f]^{\prime}=-2 f,[e, f]^{\prime}=h$. On one hand, by the proof of Lemma 3, we have that $\alpha$ is an automorphism of $\left(\mathfrak{g},[\cdot, \cdot]^{\prime}\right)$. On the other hand, by Lie theory, the automorphism of $A_{1}$ has the form $\alpha=\exp ^{a d k h}, k \in \mathbb{C}$. Thus

$$
\alpha(h, e, f)=(h, e, f)\left(\begin{array}{ccc}
1 & 0 & 0 \\
0 & \exp ^{2 k} & 0 \\
0 & 0 & \exp ^{-2 k}
\end{array}\right) .
$$

Now it follows that

$$
\begin{aligned}
& {[h, e]=\alpha\left([h, e]^{\prime}\right)=2 \exp ^{2 k} e,} \\
& {[h, f]=\alpha\left([h, f]^{\prime}\right)=-2 \exp ^{-2 k} f,} \\
& {[e, f]=\alpha\left([e, f]^{\prime}\right)=h .}
\end{aligned}
$$

Let $a=\exp ^{2 k} \neq 0$. The result follows.

\section{The Representations of Multiplicative Hom-Lie Algebras}

First we give the definition of the representations of multiplicative Hom-Lie algebras. 
Definition 5 (see [7]). Let $(\mathfrak{g},[\cdot, \cdot], \alpha)$ be a multiplicative HomLie algebra, $V$ a finite dimensional vector space, and $\beta \epsilon$ $\mathrm{gl}(V)$. If a linear map $\rho: \mathfrak{g} \rightarrow \mathrm{gl}(V)$ satisfies

$$
\begin{array}{r}
\rho([x, y]) \beta(v)=\rho(\alpha(x)) \rho(y) v-\rho(\alpha(y)) \rho(x) v, \\
\forall x, y \in \mathfrak{g}, \quad v \in V, \\
3 \beta(\rho(x) v)=\rho(\alpha(x)) \beta(v), \quad \forall x \in \mathfrak{g}, \quad v \in V,
\end{array}
$$

then $(\rho, V, \beta)$ is called a representation of $(\mathfrak{g},[\cdot, \cdot], \alpha)$, and $(V, \beta)$ is called a Hom-g-module via the action $x v=$ $\rho(x) v$, for all $x \in \mathfrak{g}, v \in V$.

For a Hom-g-module $(V, \beta)$, if a subspace $V_{1} \subseteq V$ is invariant under $\beta$, then $\left(V_{1}, \beta\right)$ is called a Hom- $\mathfrak{g}$-submodule of $(V, \beta)$. A Hom-g-module $(V, \beta)$ is called irreducible, if it has precisely two Hom-g-submodules (itself and 0 ). A Hom- $\mathfrak{g}$ module $(V, \beta)$ is called completely reducible if $V=V_{1} \oplus \cdots \oplus V_{s}$, where $\beta\left(V_{i}\right) \subseteq V_{i}(i=1, \ldots, s)$ and $\left(V_{i}, \beta\right)(i=1, \ldots, s)$ are irreducible Hom-g-submodules.

Proposition 6. Let $(\mathfrak{g},[\cdot, \cdot], \alpha)$ be a multiplicative Hom-Lie algebra with $\alpha$ invertible, $(\rho, V, \beta)$ its representation with $\beta$ invertible, and $\left(\mathfrak{g},[\cdot, \cdot]_{\mathfrak{g}}^{\prime}\right)$ the compatible Lie algebra. Let $\rho^{\prime}=$ $\beta^{-1} \rho$; then $\left(\rho^{\prime}, V\right)$ is a representation of $\left(\mathfrak{g},[\cdot, \cdot]_{\mathfrak{g}}^{\prime}\right)$.

Proof. Equation (12) is equivalent to

$$
\rho(\alpha(x))=\beta \rho(x) \beta^{-1}, \quad \forall x \in \mathfrak{g} .
$$

Equation (11) is equivalent to

$$
\begin{array}{r}
\rho[x, y]=\beta \rho(x) \beta^{-1} \rho(y) \beta^{-1}-\beta \rho(y) \beta^{-1} \rho(x) \beta^{-1}, \\
\forall x \in \mathfrak{g}, \quad v \in V .
\end{array}
$$

Denote that by $\alpha(x)=x^{\prime}$, (13) can be rewritten as $\rho\left(\alpha^{-1}\left(x^{\prime}\right)\right)=\beta^{-1} \rho\left(x^{\prime}\right) \beta$; by the arbitrary of $x$ and the invertibility of $\alpha$ we have

$$
\rho\left(\alpha^{-1}(x)\right)=\beta^{-1} \rho(x) \beta, \quad \forall x \in \mathfrak{g} .
$$

On vector space $\beta^{-1} \rho(\mathfrak{g})$, for all $x, y \in \mathfrak{g}$, define a commutator bracket $[\cdot, \cdot]^{\prime}$ as

$$
\begin{aligned}
& {\left[\beta^{-1} \rho(x), \beta^{-1} \rho(y)\right]^{\prime}} \\
& \quad=\beta^{-1} \rho(x) \beta^{-1} \rho(y)-\beta^{-1} \rho(y) \beta^{-1} \rho(x) \\
& \quad=\left[\rho^{\prime}(x), \rho^{\prime}(y)\right]^{\prime} .
\end{aligned}
$$

Clearly, $\left(\beta^{-1} \rho(\mathfrak{g}),[\cdot, \cdot]^{\prime}\right)$ is a Lie algebra.
On the other hand, for all $x, y \in \mathfrak{g}$,

$$
\begin{aligned}
\rho^{\prime}\left([x, y]_{\mathfrak{g}}^{\prime}\right)= & \rho^{\prime}\left(\alpha^{-1}([x, y])\right) \\
= & \beta^{-1} \rho\left(\left[\alpha^{-1}(x), \alpha^{-1}(y)\right]\right) \\
= & \beta^{-1}\left(\beta \rho\left(\alpha^{-1}(x)\right) \beta^{-1} \rho\left(\alpha^{-1}(y)\right) \beta^{-1}\right. \\
& \left.\quad-\beta \rho\left(\alpha^{-1}(y)\right) \beta^{-1} \rho\left(\alpha^{-1}(x)\right) \beta^{-1}\right) \\
= & \beta^{-1} \rho(x) \beta \beta^{-1} \beta^{-1} \rho(y) \beta \beta^{-1} \\
& -\beta^{-1} \rho(y) \beta \beta^{-1} \beta^{-1} \rho(x) \beta \beta^{-1} \\
= & \beta^{-1} \rho(x) \beta^{-1} \rho(y)-\beta^{-1} \rho(y) \beta^{-1} \rho(x) \\
= & {\left[\rho^{\prime}(x), \rho^{\prime}(y)\right]^{\prime} . }
\end{aligned}
$$

Then the result follows easily.

From Proposition 6, we can get a method of computing representations of a multiplicative Hom-Lie algebra with $\alpha, \beta$ invertible.

Let $(\rho, V, \beta)$ be a representation of a Lie-type Hom-Lie algebra. If $\rho=\beta \rho^{\prime}$, where $\left(\rho^{\prime}, V\right)$ is a representation of the compatible Lie algebra, then $(\rho, V, \beta)$ is called a Lie-type representation. It is easy to know that the representation in Proposition 6 is Lie-type. In addition, suppose that $\left(\rho^{\prime}, V\right)$ is an irreducible representation of the compatible Lie algebra; then $(\rho, V, \beta)$ is called a regular Lie-type representation.

Theorem 7. Let $(\mathfrak{g},[\cdot, \cdot], \alpha)$ be a Lie-type Hom-Lie algebra with the compatible Lie algebra $\left(\mathfrak{g},[\cdot, \cdot]^{\prime}\right)$.

(1) If $(\rho, V, \beta)$ ( $\beta$ invertible) is a representation of $(\mathfrak{g},[\cdot, \cdot], \alpha)$, then

$$
\beta\left(\rho^{\prime}(x) v\right)=\rho^{\prime}(\alpha(x)) \beta(v), \quad \forall x \in \mathfrak{g}, \quad v \in V,
$$

where $\left(\rho^{\prime}, V\right)=\left(\beta^{-1} \rho, V\right)$ is a representation of the compatible Lie algebra.

(2) Suppose that $\left(\rho^{\prime}, V\right)$ is a representation of $\left(\mathfrak{g},[\cdot, \cdot]^{\prime}\right)$. If $\exists \beta \in g l(V)$ such that (18) is satisfied, let $\rho=\beta \rho^{\prime}$; then $(\rho, V, \beta)$ is a representation of $(\mathfrak{g},[\cdot, \cdot], \alpha)$.

Proof. (1) By the invertibility of $\beta$ and (12), we can get (18) easily.

(2) for all $x \in \mathfrak{g}, v \in V$, (12) follows from (18) easily. for all $x, y \in \mathfrak{g}, v \in V$, we have

$$
\begin{aligned}
\rho( & {[x, y]) \beta(v)=\beta\left(\rho^{\prime}\left(\alpha\left([x, y]^{\prime}\right)\right) \beta(v)\right) } \\
& =\beta\left(\left(\rho^{\prime}(\alpha(x)) \rho^{\prime}(\alpha(y))-\rho^{\prime}(\alpha(y)) \rho^{\prime}(\alpha(x))\right) \beta(v)\right) \\
& =\beta\left(\rho^{\prime}(\alpha(x)) \beta\left(\rho^{\prime}(y) v\right)-\rho^{\prime}(\alpha(y)) \beta\left(\rho^{\prime}(x) v\right)\right) \\
& =\rho(\alpha(x)) \rho(y) v-\rho(\alpha(x)) \rho(y) v .
\end{aligned}
$$

Now we can get (11). Therefore $(\rho, V, \beta)$ is a representation of $(\mathfrak{g},[\cdot, \cdot], \alpha)$. 


\section{Regular Lie-Type Representations of 3-Dimensional Simple Multiplicative Hom-Lie Algebras}

Lemma 8. Let $(\rho, V, \beta)$ be a representation of a multiplicative Hom-Lie algebra $(\mathfrak{g},[\cdot, \cdot], \alpha)$; then $(\operatorname{Ker}(\beta), \beta)$ is a Hom-gsubmodule of $(V, \beta)$.

Proof. for all $v \in \operatorname{Ker}(\beta)$, by $\beta(\rho(x) v)=\rho(\alpha(x)) \beta(v)=$ 0 , for all $x \in \mathfrak{g}$, it is easy to know that $\rho(x) \operatorname{Ker}(\beta) \subseteq \operatorname{Ker}(\beta)$; then the result follows easily.

Lemma 9. Let $(\rho, V, \beta)$ be an irreducible or a completely reducible representation of a multiplicative Hom-Lie algebra $(\mathfrak{g},[\cdot, \cdot], \alpha)$; then $\beta$ is invertible.

Proof. By the reason of Lemma 8 , if $(V, \beta)$ is an irreducible Hom-g module, we have that $\beta$ is invertible. If $(V, \beta)$ is a completely reducible Hom-g module, then $V=V_{1} \oplus \cdots \oplus$ $V_{s}$, where $\left(V_{i},\left.\beta\right|_{V_{i}}\right)(i=1, \ldots, s)$ are irreducible Hom-g submodules. By the irreducibility of $\left(V_{i},\left.\beta\right|_{V_{i}}\right)$, we have that $\left.\beta\right|_{V_{i}}(i=1, \ldots, s)$ is invertible, so $\beta$ is an invertible linear map of $V$.

Lemma 10. Let $(\rho, V, \beta)$ with $\beta$ invertible be a nontrivial finite dimensional regular Lie-type representation of a Lie-type HomLie algebra; then $(V, \beta)$ is an irreducible Hom-g-module.

Proof. Suppose to the contrary that $(V, \beta)$ is reducible. Then we assume that $\left(V_{1}, \beta\right)$ is a nontrivial Hom-g-submodule of $(V, \beta)$. Let $\left(\rho^{\prime}, V\right)=\left(\beta^{-1} \rho, V\right)$ be the representation of the compatible Lie algebra. Then

$$
\rho^{\prime}(x) V_{1}=\beta^{-1}\left(\rho(x) V_{1}\right) \subseteq \beta^{-1}\left(V_{1}\right) \subseteq V_{1} .
$$

That is, $\left(\rho^{\prime}, V_{1}\right)$ is a nontrivial subrepresentation of the compatible Lie algebra, which is a contradiction. Therefore $(V, \beta)$ is an irreducible Hom- $\mathfrak{g}$-module.

It is natural to ask the question: are there nontrivial finite dimensional regular Lie-type representations in 3dimensional simple multiplicative Hom-Lie algebras? Let us see the following theorem.

Theorem 11. Let $(\mathfrak{g},[\cdot, \cdot], \alpha)$ be a 3-dimensional simple multiplicative Hom-Lie algebra; then there exist nontrivial finite dimensional regular Lie-type representations $(\rho, V, \beta)$, and these representations are irreducible. For every such representation, $\rho(h)$ is a semisimple linear transformation of $V$. In addition, there is a basis $\left\{v_{0}, v_{1}, \ldots, v_{m}\right\}$ of $V$ such that

(a) $\rho(h) v_{i}=(m-2 i) a^{-i} b_{0} v_{i}, \beta\left(v_{i}\right)=a^{-i} b_{0} v_{i}, i=0, \ldots, m$;

(b) $\rho(f) v_{i}=(i+1) a^{-i-1} b_{0} v_{i+1}, \quad i=0, \ldots, m-$ $1 ; \rho(f) v_{m}=0$;

(c) $\rho(e) v_{0}=0, \rho(e) v_{i}=(m-i+1) a^{-i+1} b_{0} v_{i-1}, i=1, \ldots, m$.

Proof. Let $\left(\rho^{\prime}, V\right)$ be an $m+1$ dimensional irreducible representation of the compatible Lie algebra $A_{1}$. Take $\left\{v_{0}, v_{1}, \ldots, v_{m}\right\}$ as a basis of $V$, where $v_{i}=1 / i \rho^{\prime}(f) v_{i-1}(i=$ $1, \ldots, m)$ satisfies

$$
\begin{gathered}
\rho^{\prime}(h) v_{i}=(m-2 i) v_{i}, \\
\rho^{\prime}(e) v_{i}=(m-i+1) v_{i-1} \quad(i=1, \ldots, m), \\
\rho^{\prime}(e) v_{0}=0 .
\end{gathered}
$$

Now we prove that when $\beta \in \operatorname{gl}(V)$ is defined by

$$
\beta\left(\begin{array}{c}
v_{0} \\
v_{1} \\
\vdots \\
v_{m}
\end{array}\right)^{T}=\left(\begin{array}{c}
v_{0} \\
v_{1} \\
\vdots \\
v_{m}
\end{array}\right)^{T}\left(\begin{array}{cccc}
b_{0} & & & \\
& a^{-1} b_{0} & \\
& & \ddots & \\
& & & a^{-m} b_{0}
\end{array}\right),
$$

(18) is always satisfied. Because

$$
\begin{aligned}
\beta\left(\rho^{\prime}(h) v_{i}\right)=( & m-2 i) \beta\left(v_{i}\right)=(m-2 i) a^{-i} b_{0} v_{i}, \\
\rho^{\prime}(\alpha(h)) \beta\left(v_{i}\right)= & \rho^{\prime}(h) a^{-i} b_{0} v_{i} \\
= & a^{-i} b_{0}(m-2 i) v_{i}=\beta\left(\rho^{\prime}(h) v_{i}\right), \\
& i=0, \ldots, m, \\
\beta\left(\rho^{\prime}(f) v_{i}\right)=(i+1) \beta\left(v_{i+1}\right)=(i+1) a^{-i-1} b_{0} v_{i+1}, & \\
\rho^{\prime}(\alpha(f)) \beta\left(v_{i}\right) & =a^{-1} \rho^{\prime}(f) a^{-i} b_{0} v_{i} \\
& =a^{-i-1} b_{0}(i+1) v_{i+1} \\
& =\beta\left(\rho^{\prime}(f) v_{i}\right), \quad i=0, \ldots, m, \\
\beta\left(\rho^{\prime}(e) v_{i}\right) & =\beta\left((m-i+1) v_{i-1}\right) \\
& =(m-i+1) \beta\left(v_{i-1}\right) \\
& =(m-i+1) a^{-(i-1)} b_{0} v_{i-1}, \\
& =a^{-(i-1)} b_{0}(m-i+1) v_{i-1} \\
\rho^{\prime}(\alpha(e)) \beta\left(v_{i}\right) & =a \rho^{\prime}(e) \beta\left(v_{i}\right) \\
& \left.=\rho^{\prime}(e) a^{-(i-1)} b_{0} v_{i}\right), \quad i=0, \ldots, m .
\end{aligned}
$$

Thus for $\beta$ defined by (22), (18) is always established. Let $\rho=\beta \rho^{\prime}$; then it follows from Theorem 7 (2) that $(\rho, V, \beta)$ is a nontrivial finite dimensional regular Lie-type representation of $(\mathfrak{g},[\cdot, \cdot], \alpha)$. By Lemma 10, we have that $(\rho, V, \beta)$ is irreducible.

Furthermore, we have

$$
\begin{array}{r}
\rho(h) v_{i}=\beta\left(\rho^{\prime}(h) v_{i}\right)=(m-2 i) a^{-i} b_{0} v_{i}, \\
i=0, \ldots, m,
\end{array}
$$


and thus we can get (a). For some fixed $b_{0}$, it is obvious that $\rho(h)$ is a semisimple linear transformation of $V$. Take $x=e, f$ in $\rho(x) v_{i}=\beta\left(\rho^{\prime}(x) v_{i}\right)(i=0, \ldots, m)$; we can get (b) and (c) easily.

\section{Irreducible and Completely Reducible Representations of a 3-Dimensional Simple Multiplicative Hom-Lie Algebra}

In this section, $(\mathfrak{g},[\cdot, \cdot], \alpha)$ denotes a 3 -dimensional simple multiplicative Hom-Lie algebra.

By Theorem 11, we know that there exist nontrivial finite dimensional irreducible regular Lie-type representations of $(\mathfrak{g},[\cdot, \cdot], \alpha)$. However, are there other nontrivial irreducible representations of $(\mathfrak{g},[\cdot, \cdot], \alpha)$ ? Is any finite dimensional representation of $(\mathfrak{g},[\cdot, \cdot], \alpha)$ completely reducible? We will study these questions in this section.

By Lemma 9, we only need to consider the case when $\beta$ is invertible.

Let $(\rho, V, \beta)$ with $\beta$ invertible be a finite dimensional representation of $(\mathfrak{g},[\cdot, \cdot], \alpha)$. By Proposition 6 , we have that $(\rho, V, \beta)$ is of Lie-type, and $\left(\rho^{\prime}, V\right)=\left(\beta^{-1} \rho, V\right)$ is a finite dimensional representation of the compatible Lie algebra $A_{1}$. By Weyl theorem, we know that $\left(\rho^{\prime}, V\right)$ is completely reducible. That is, $V=V_{1} \oplus \cdots \oplus V_{s}$, where $V_{i}(i=1, \ldots, s)$ are irreducible $A_{1}$-modules. Suppose that $\operatorname{dim} V_{i}=m_{i}+1$. Denote that a tuple $\tau=\left(m_{1}, \ldots, m_{s}\right)$. By the representation theory of $A_{1}$, we have that $V_{j}(j=1, \ldots, s)$ is a highest weight module with highest weight vectors $v_{j 0}$ and the highest weight $m_{j}$, respectively. Take $\left\{v_{j 0}, v_{j 1}, \ldots, v_{j m_{j}}\right\}$ as a basis of $V_{j}$ satisfying

$$
\begin{gathered}
v_{j i}=\frac{1}{i \rho^{\prime}(f) v_{j, i-1}}, \quad\left(i=1, \ldots, m_{j}\right), \\
\rho^{\prime}(h) v_{j i}=\left(m_{j}-2 i\right) v_{j i}, \quad\left(i=0, \ldots, m_{j}\right), \\
\rho^{\prime}(e) v_{j i}=\left(m_{j}-i+1\right) v_{j, i-1}, \quad\left(i=1, \ldots, m_{j}\right), \\
\rho^{\prime}(e) v_{0}=0 .
\end{gathered}
$$

Theorem 12. When $\tau=\left(m_{1}, \ldots, m_{s}\right), m_{i} \neq m_{j}$ for $1 \leq i \neq j \leq$ $s$, then

(1) $\beta=\operatorname{diag}\left(\ldots, b_{i 0}, b_{i 0} / a, \ldots, b_{i 0} / a^{m_{i}}, \ldots\right), i=1, \ldots, s$;

(2) $(V, \beta)$ is a completely reducible Hom-g-module with a decomposition $V=V_{1} \oplus \cdots \oplus V_{s}$, where $\left(V_{i}, \beta_{i}\right), \quad(i=$ $1, \ldots, s)$ are irreducible Hom-g-submodules; here $\beta_{i}=$ $\left.\beta\right|_{V_{i}}$

Proof. (1) According to (18), we get the result.

(2) Because $\left(V_{j}\right) \subseteq V_{j}, j=1, \ldots, s$,

$$
\rho(x) V_{j}=\beta\left(\rho^{\prime}(x) V_{j}\right) \subseteq V_{j} .
$$

Let $\beta_{j}=\left.\beta\right|_{V_{j}}$, combined with Lemma 10; we have that $\left(V_{j}, \beta_{j}\right)(j=1, \ldots, s)$ are irreducible Hom-g-submodules.
When $\tau=(m, \ldots, m)$, by $(18)$ it can be checked that

$$
\beta=\left(\begin{array}{cccc}
B_{11} & B_{12} & \ldots & B_{1 s} \\
B_{21} & B_{22} & \ldots & B_{2 s} \\
\vdots & \vdots & \ddots & \vdots \\
B_{s 1} & B_{s 2} & \ldots & B_{s s}
\end{array}\right),
$$

where $B_{i j}=\operatorname{diag}\left(b_{i j}, b_{i j} / a, \ldots, b_{i j} / a^{m}\right)$.

Take

$$
B_{1}=\left(\begin{array}{cccc}
b_{11} & b_{12} & \ldots & b_{1 s} \\
b_{21} & b_{22} & \ldots & b_{2 s} \\
\vdots & \vdots & \ddots & \vdots \\
b_{s 1} & b_{s 2} & \ldots & b_{s s}
\end{array}\right)
$$

By the theory of linear algebra, there is an invertible matrix

$$
P=\left(\begin{array}{cccc}
p_{11} & p_{12} & \cdots & p_{1 s} \\
p_{21} & p_{22} & \cdots & p_{2 s} \\
\vdots & \vdots & \ddots & \vdots \\
p_{s 1} & p_{s 2} & \cdots & p_{s s}
\end{array}\right)
$$

such that $P^{-1} B_{1} P$ is a Jordan canonical form; that is,

$$
P^{-1} B_{1} P=\left(\begin{array}{cccc}
J_{11} & & & \\
& J_{22} & & \\
& & \ddots & \\
& & & J_{t t}
\end{array}\right) \text {, }
$$

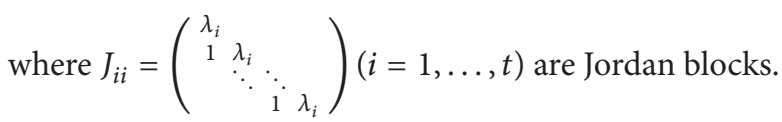

Theorem 13. The condition is the same as the previous remark.

(1) If $t=1$, then $(V, \beta)$ is a reducible but not completely reducible Hom-g-module.

(2) If $s>t>1$, then $(V, \beta)$ is a reducible but not completely reducible Hom- $\mathfrak{g}$-module.

(3) If $t=s$, then $(V, \beta)$ is a completely reducible Hom- $\mathfrak{g}$ module.

Proof. Let

$$
\begin{aligned}
& v_{i 0}^{\prime}=p_{1 i} v_{10}+p_{2 i} v_{20}+\cdots+p_{s i} v_{s 0}, \quad i=1, \ldots, s, \\
& v_{i j}^{\prime}=\frac{1}{j} \rho^{\prime}(f) v_{i, j-1}^{\prime}, \quad i=1, \ldots, s ; j=1, \ldots, m .
\end{aligned}
$$

That is,

$$
\begin{array}{r}
v_{i j}^{\prime}=p_{1 i} v_{1 j}+p_{2 i} v_{2 j}+\cdots+p_{s i} v_{s j}, \\
i=1, \ldots, s ; j=1, \ldots, m .
\end{array}
$$

Then

$$
\begin{aligned}
& \left(v_{10}^{\prime}, \ldots, v_{1 m}^{\prime}, \ldots, v_{s 0}^{\prime}, \ldots, v_{s m}^{\prime}\right) \\
& \quad=\left(v_{10}, \ldots, v_{1 m}, \ldots, v_{s 0}, \ldots, v_{s m}\right) Q
\end{aligned}
$$


where

$$
Q=\left(\begin{array}{cccc}
Q_{11} & Q_{12} & \ldots & Q_{1 s} \\
Q_{21} & Q_{22} & \ldots & Q_{2 s} \\
\vdots & \vdots & \ddots & \vdots \\
Q_{s 1} & Q_{s 2} & \ldots & Q_{s s}
\end{array}\right)
$$

here $Q_{i j}$ are $m \times m$ matrices satisfying

$$
Q_{i j}=\operatorname{diag}\left(p_{i j}, \ldots, p_{i j}\right), \quad i, j=1, \ldots, s .
$$

Because $P$ is invertible, it is easy to check that the matrix $Q$ is invertible; therefore $\left\{v_{10}^{\prime}, \ldots, v_{1 m}^{\prime}, \ldots, v_{s 0}^{\prime}, \ldots, v_{s m}^{\prime}\right\}$ is a basis of $V$.

Let $V_{i}^{\prime}=\mathbb{C}\left\{v_{i 0}^{\prime}, v_{i 1}^{\prime}, \ldots, v_{i m}^{\prime}\right\}$, because

$$
\rho^{\prime}(h) v_{i 0}^{\prime}=\rho^{\prime}(h)\left(p_{1 i} v_{10}+p_{2 i} v_{20}+\cdots+p_{s i} v_{s 0}\right)=m v_{i 0} \text {. }
$$

By (32) and the representation of Lie algebra $A_{1}$, we have

$$
\begin{aligned}
& \rho^{\prime}(h)\left(v_{i 0}^{\prime}, \ldots, v_{i m}^{\prime}\right)=\left(v_{i 0}^{\prime}, \ldots, v_{i m}^{\prime}\right)\left(\begin{array}{cccc}
m & & & \\
& m-2 & & \\
& & \ddots & \\
& & & -m
\end{array}\right) \text {, } \\
& \rho^{\prime}(f)\left(v_{i 0}^{\prime}, \ldots, v_{i m}^{\prime}\right)=\left(v_{i 0}^{\prime}, \ldots, v_{i m}^{\prime}\right)\left(\begin{array}{ccccc}
0 & 0 & \ldots & 0 & 0 \\
1 & 0 & \ldots & 0 & 0 \\
0 & 2 & \ldots & 0 & 0 \\
\vdots & \vdots & \ddots & \vdots & \vdots \\
0 & 0 & \ldots & m & 0
\end{array}\right) \text {, } \\
& \rho^{\prime}(e)\left(v_{i 0}^{\prime}, \ldots, v_{i m}^{\prime}\right)=\left(v_{i 0}^{\prime}, \ldots, v_{i m}^{\prime}\right) \\
& \times\left(\begin{array}{ccccc}
0 & m & 0 & \ldots & 0 \\
0 & 0 & m-1 & \ldots & 0 \\
\vdots & \vdots & \vdots & \ddots & \vdots \\
0 & 0 & 0 & \ldots & 1 \\
0 & 0 & 0 & \ldots & 0
\end{array}\right)
\end{aligned}
$$

Through (38) it is easy to get that $V_{1}^{\prime}, \ldots, V_{s}^{\prime}$ are $A_{1}$ irreducible-modules.

(1) In this case, by (30), we have

$$
\beta\left(v_{10}^{\prime}, v_{20}^{\prime}, \ldots, v_{s 0}^{\prime}\right)=\left(v_{10}^{\prime}, v_{20}^{\prime}, \ldots, v_{s 0}^{\prime}\right)\left(\begin{array}{cccc}
\lambda & 0 & & 0 \\
1 & \lambda & & \\
& \ddots & \ddots & \\
& & 1 & \lambda
\end{array}\right) .
$$

Let

$$
V_{i}^{\prime}=\mathbb{C}\left\{v_{i 0}^{\prime}, \ldots, v_{i m}^{\prime}\right\}, \quad i=1, \ldots, s,
$$

By (32), it is easy to check that

$$
\beta\left(V_{i}^{\prime}\right) \subseteq V_{i}^{\prime}+V_{i+1}^{\prime}, \quad i=1, \ldots, s-1 ; \beta\left(V_{s}^{\prime}\right) \subseteq V_{s}^{\prime} .
$$

Then $\rho(x) V_{s}^{\prime}=\beta\left(\rho^{\prime}(x) V_{s}^{\prime}\right) \subseteq V_{s}^{\prime}$. Let $\beta_{s}=\left.\beta\right|_{V_{s}^{\prime}}$; then $\left(V_{s}^{\prime}, \beta_{s}\right)$ is an irreducible Hom-g-submodule, but $(V, \beta)$ is not completely reducible.

(2) Suppose (omit the order of $v_{10}^{\prime}, \ldots, v_{s 0}^{\prime}$ )

$$
\beta\left(v_{10}^{\prime}, v_{20}^{\prime}, \ldots, v_{s 0}^{\prime}\right)
$$

$$
=\left(v_{10}^{\prime}, v_{20}^{\prime}, \ldots, v_{s 0}^{\prime}\right)\left(\begin{array}{cccccc}
\lambda_{1} & & & & & \\
& \ddots & & & & \\
& & \lambda_{j} & & & \\
& & & B_{j+1} & & \\
& & & & \ddots & \\
& & & & & B_{t}
\end{array}\right) ;
$$

here $\lambda_{i} \in \mathbb{C}, i=1, \ldots, j, B_{j+1}, \ldots, B_{t}$ are $k_{p} \times k_{p}$ nondiagonal Jordan blocks $\left(k_{p} \in \mathbb{Z}^{+}, p=1, \ldots, t-j\right)$.

Let

$$
V_{i}^{\prime}=\mathbb{C}\left\{v_{i 0}^{\prime}, \ldots, v_{i m}^{\prime}\right\}, \quad i=1, \ldots, j,
$$

denoted by $\beta_{i}=\left.\beta\right|_{V_{i}^{\prime}}$. It is easy to check that

$$
\begin{gathered}
\beta_{i}\left(V_{i}^{\prime}\right) \subseteq V_{i}^{\prime}, \\
\rho(x) V_{i}^{\prime}=\beta\left(\rho^{\prime}(x) V_{i}^{\prime}\right) \subseteq \beta\left(V_{i}^{\prime}\right) \subseteq V_{i}^{\prime}, \quad i=1, \ldots, j .
\end{gathered}
$$

By the statement of the proof and Lemma 10, we have that $\left(V_{i}^{\prime}, \beta_{i}\right)(i=1, \ldots, j)$ are irreducible Hom- $\mathfrak{g}$-submodules.

Let

$$
\begin{gathered}
V_{j+p}^{\prime}=\mathbb{C}\left\{v_{j+k_{1}+\cdots+k_{p-1}+1,0}^{\prime}, \ldots,\right. \\
v_{j+k_{1}+\cdots+k_{p-1}+1, m}^{\prime}, \ldots, \\
v_{j+k_{1}+\cdots+k_{p}, 0}^{\prime}, \ldots, \\
\left.v_{j+k_{1}+\cdots+k_{p}, m}^{\prime}\right\}, \\
p=1, \ldots, t-j .
\end{gathered}
$$

Then

$$
\beta\left(V_{j+p}^{\prime}\right) \subseteq V_{j+p}^{\prime}, \quad p=1, \ldots, t-j .
$$

Let $\beta_{i}=\left.\beta\right|_{V_{i}} ^{\prime}, \quad(i=j+1, \ldots, t)$; then $\left(V_{i}, \beta_{i}\right)(i=j+1, \ldots, t)$ are Hom-g-submodules. As (1) of the theorem, we can prove that $\left(V_{i}, \beta_{i}\right)(i=j+1, \ldots, t)$ are reducible but not completely reducible Hom- $\mathfrak{g}$-modules. We have the conclusion. 
(3) In this case, by (30) and Theorem 12 (1) we get

$$
\begin{aligned}
& \beta\left(\begin{array}{c}
v_{10}^{\prime} \\
\cdots \\
v_{1 m}^{\prime} \\
\cdots \\
v_{s 0}^{\prime} \\
\cdots \\
v_{s m}^{\prime}
\end{array}\right)^{t}
\end{aligned}
$$

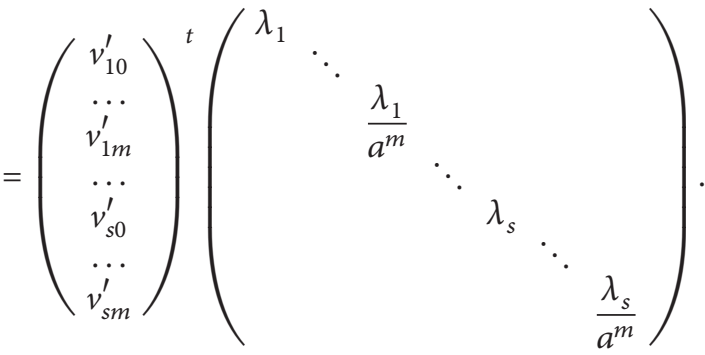

Let

$$
V_{i}^{\prime}=\mathbb{C}\left\{v_{i 0}^{\prime}, \ldots, v_{i m}^{\prime}\right\}, \quad i=1, \ldots, s
$$

then $V_{1}^{\prime}, \ldots, V_{s}^{\prime}$ are irreducible $A_{1}$-modules and

$$
\begin{gathered}
\beta\left(V_{i}^{\prime}\right) \subseteq V_{i}^{\prime}, \quad i=1, \ldots, s, \\
\rho(x) V_{i}^{\prime}=\beta\left(\rho^{\prime}(x) V_{i}^{\prime}\right) \subseteq V_{i}^{\prime}, \quad i=1, \ldots, s, \forall x \in \mathfrak{g} .
\end{gathered}
$$

Denoting by $\beta_{i}=\left.\beta\right|_{V_{i}}, i=1, \ldots, s$, thanks to Lemma 10 we have that $\left(V_{i}^{\prime}, \beta_{i}\right)(i=1, \ldots, s)$ are irreducible Hom$\mathfrak{g}$-submodules. So $(V, \beta)$ is a completely reducible Hom- $\mathfrak{g}$ module.

When $\tau=\left(m_{1}, \ldots, m_{1}, \ldots, m_{i}, \ldots, m_{i}, m_{i+1}, \ldots, m_{l}\right)$, suppose that the multiplicity of $m_{t}(t=1, \ldots, i)$ is $k_{t}$ and $k_{1}+\cdots+k_{i}+l-i=s$. By (18) we can get $\beta$ as follows:

$$
\begin{aligned}
& \beta\left(\begin{array}{c}
v_{10} \\
\cdots \\
v_{1 m_{1}} \\
\cdots \\
v_{s 0} \\
\cdots \\
v_{s m_{l}}
\end{array}\right) \\
& =\left(\begin{array}{c}
v_{10} \\
\cdots \\
v_{1 m_{1}} \\
\cdots \\
v_{s 0} \\
\cdots \\
v_{s m_{l}}
\end{array}\right)\left(\begin{array}{cccccc}
B_{1} & & & & & \\
& \ddots & & & & \\
& & B_{i} & & & \\
& & & B_{i+1} & & \\
& & & & \ddots & \\
& & & & & B_{l}
\end{array}\right) \text {, }
\end{aligned}
$$

where $B_{1}, \ldots, B_{i}$ are matrices of the form (27),

$$
B_{j}=\operatorname{diag}\left(\lambda_{j}, \frac{\lambda_{j}}{a}, \ldots, \frac{\lambda_{j}}{a^{m_{j}}}\right), \quad j=i+1, \ldots, l .
$$

Theorem 14. The condition is as the previous remark. $(V, \beta)$ is completely reducible if there exist invertible matrices $P_{j}(j=$ $1, \ldots, i)$ such that $P_{j}^{-1} B_{j} P_{j}(j=1, \ldots, i)$ are diagonal matrices. Otherwise $(V, \beta)$ is reducible but not completely reducible.

Proof. It can be got from Theorems 12 and 13 directly.

Proposition 15. Let $(\rho, V, \beta)$ be a finite dimensional representation of $(\mathfrak{g},[\cdot, \cdot], \alpha)$; then $(\rho, V, \beta)$ is not always completely reducible, and $(\rho, V, \beta)$ is irreducible if and only if it is of regular Lie-type.

Proof. The claim follows from Theorems 11, 12, 13, and 14 directly.

\section{References}

[1] J. T. Hartwig, D. Larsson, and S. D. Silvestrov, "Deformations of Lie algebras using $\sigma$-derivations," Journal of Algebra, vol. 295, no. 2, pp. 314-361, 2006.

[2] D. Larsson and S. D. Silvestrov, "Quasi-hom-Lie algebras, central extensions and 2-cocycle-like identities," Journal of Algebra, vol. 288, no. 2, pp. 321-344, 2005.

[3] D. Larsson and S. D. Silvestrov, "Quasi-Lie algebras," in Noncommutative Geometry and Representation Theory in Mathematical Physics, vol. 391 of Contemporary Mathematics, pp. 241248, American Mathematical Society, Providence, RI, USA, 2005.

[4] J. Arnlind, A. Makhlouf, and S. Silvestrov, "Ternary HomNambu-Lie algebras induced by Hom-Lie algebras," Journal of Mathematical Physics, vol. 51, Article ID 043515, 2010.

[5] F. Ammar, S. Mabrouk, and A. Makhlouf, "Representations and cohomology of $n$-ary multiplicative Hom-Nambu-Lie algebras," Journal of Geometry and Physics, vol. 61, no. 10, pp. 1898-1913, 2011.

[6] Q. Jin and X. Li, "Hom-Lie algebra structures on semi-simple Lie algebras," Journal of Algebra, vol. 319, no. 4, pp. 1398-1408, 2008.

[7] Y. Sheng, "Representations of hom-Lie algebras," Algebras and Representation Theory, vol. 15, no. 6, pp. 1081-1098, 2012.

[8] D. Yau, "Hom-algebras and homology," Journal of Lie Theory, vol. 19, no. 2, pp. 409-421, 2009.

[9] J. E. Humphreys, Introduction to Lie Algebras and Representation Theory, vol. 9, Springer, New York, NY, USA, 1972.

[10] N. Jacobson, Lie Algebras, Interscience Tracts in Pure and Applied Mathematics, No. 10, Interscience, New York, NY, USA, 1962.

[11] V. G. Kac, Infinite-Dimensional Lie Algebras, Cambridge University Press, Cambridge, UK, 3rd edition, 1990. 


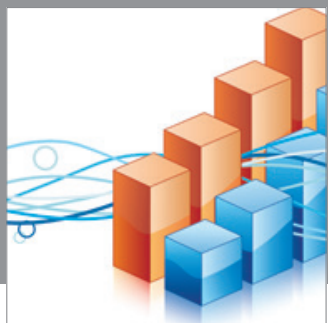

Advances in

Operations Research

mansans

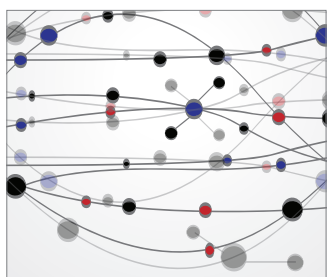

The Scientific World Journal
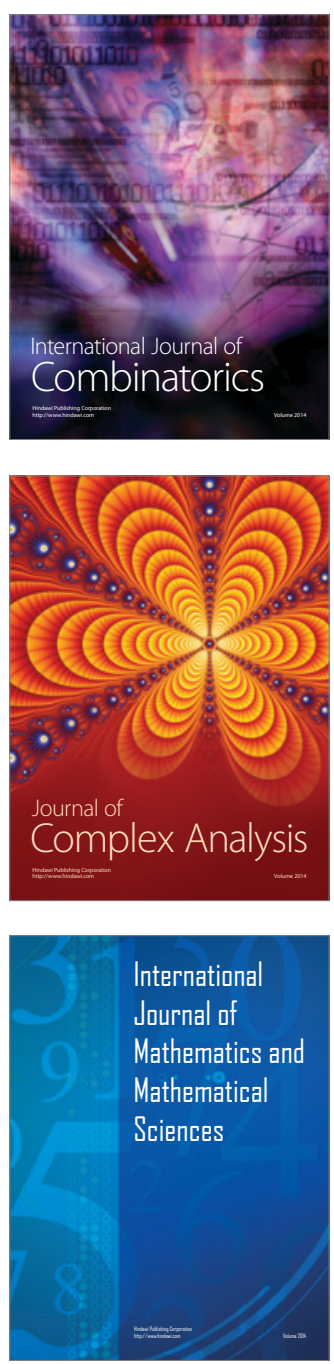
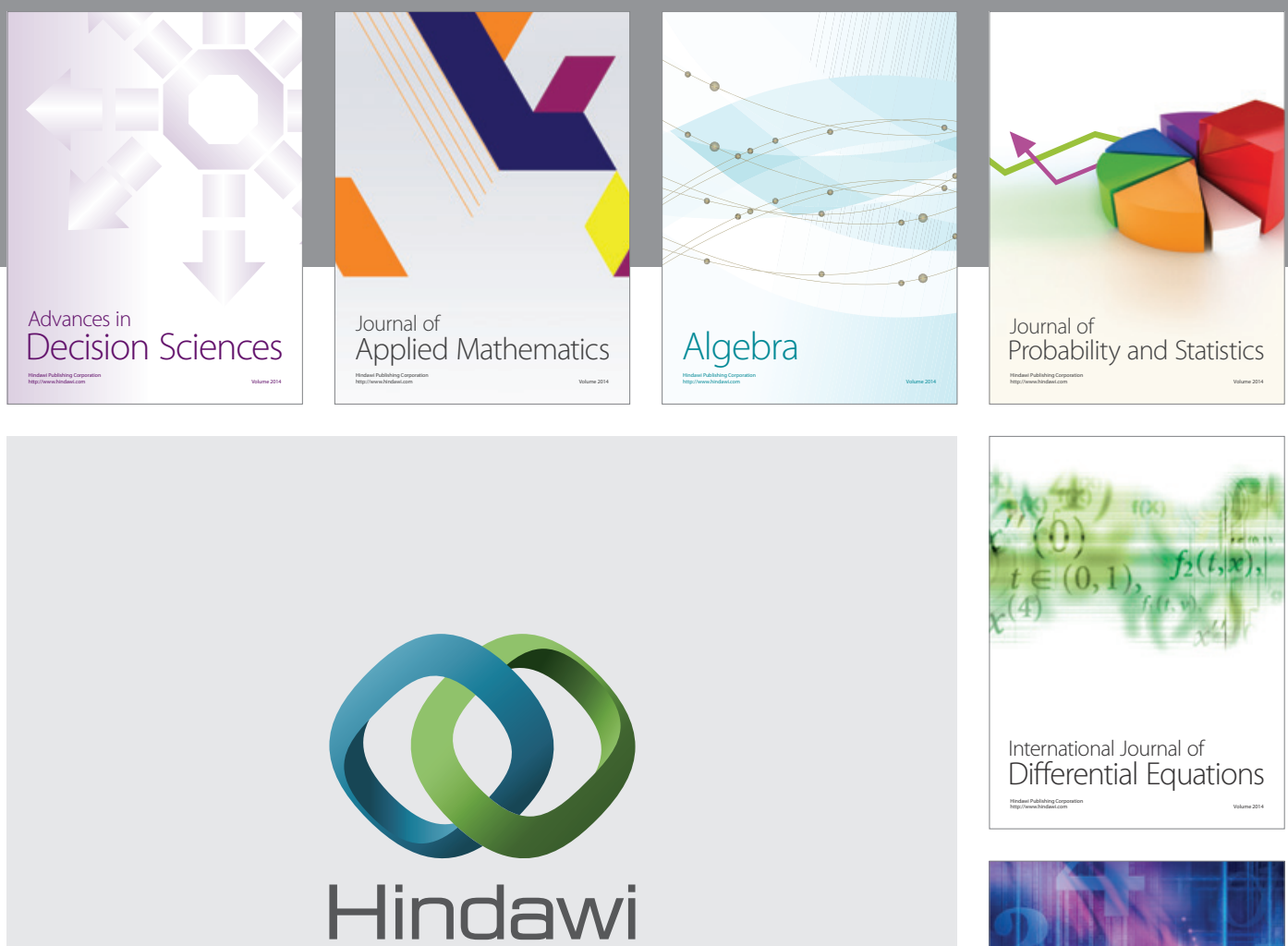

Submit your manuscripts at http://www.hindawi.com
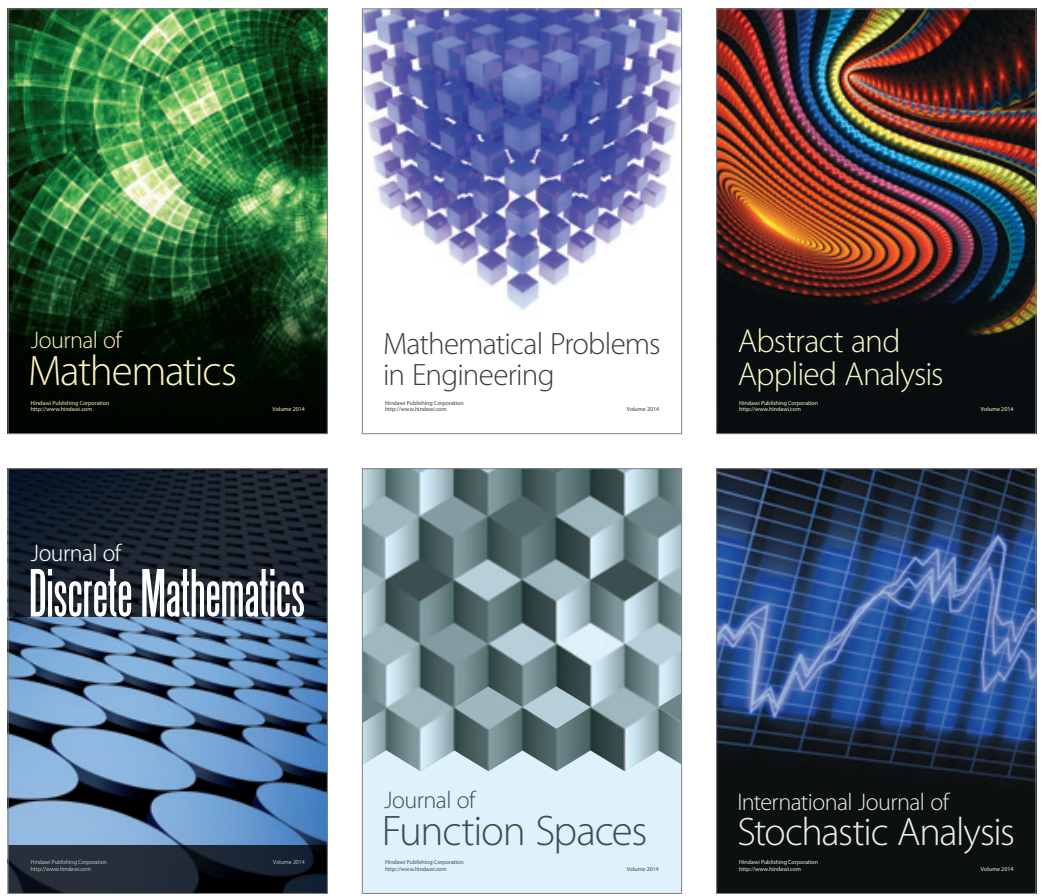

Journal of

Function Spaces

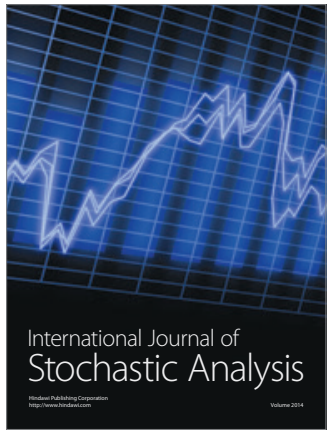

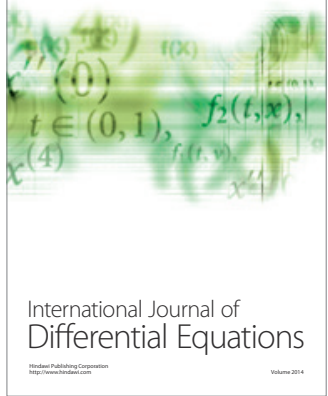
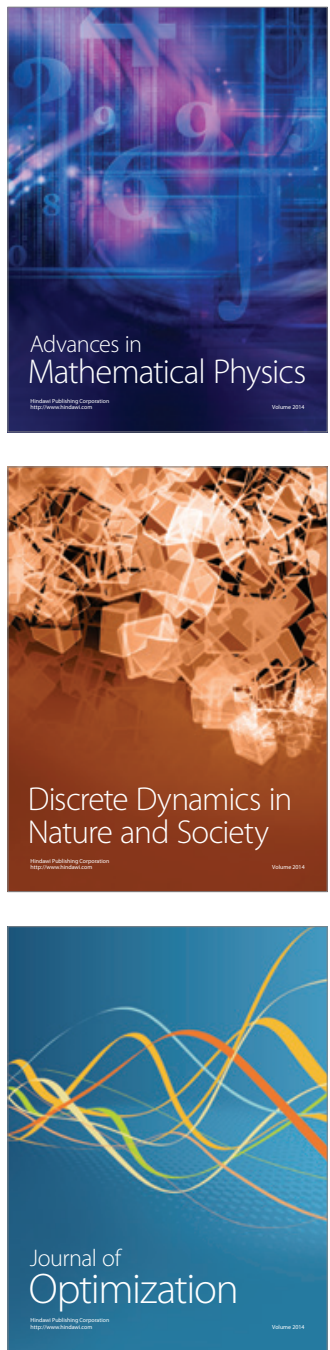